\title{
REVIEW
}

\section{Echocardiographic assessment of pulmonary hypertension: standard operating procedure}

\author{
Luke S. Howard*, Julia Grapsa*, David Dawson", Michael Bellamy", \\ John B. Chambers ", Navroz D. Masani ${ }^{+}$, Petros Nihoyannopoulos ${ }^{\#}$ \\ and J. Simon R. Gibbs*
}

\begin{abstract}
Patients with suspected pulmonary hypertension $(\mathrm{PH})$ should be evaluated using a multimodality approach to ensure that they receive a correct diagnosis. The series of investigations required includes clinical evaluation, noninvasive imaging techniques and right heart catheterisation (considered to be the "gold standard" for the diagnosis of PH). Current guidelines recommend that a detailed echocardiographic assessment is performed in all patients with suspected $\mathrm{PH}$.

In this review we summarise a protocol adopted by the National Pulmonary Hypertension Centres of UK and Ireland and approved by the British Society of Echocardiography for the evaluation of these patients. The views and measurements described are recommended for diagnosis, assisting in prognosis and providing a noninvasive means of following disease progression or response to therapy.
\end{abstract}

KEYWORDS: Diagnosis, echocardiography, prognosis, pulmonary hypertension, right ventricle

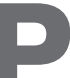

ulmonary hypertension $(\mathrm{PH})$ is defined as a haemodynamic and pathophysiological condition characterised by an increase in mean pulmonary arterial pressure $(\mathrm{Ppa})$ to $\geqslant 25 \mathrm{mmHg}$ at rest as measured by right heart catheterisation (RHC) [1]. PH may be associated with a number of conditions displaying a range of different pathological and clinical features, despite the comparable elevations in $P$ pa. The clinical classification of $\mathrm{PH}$ aims to group together different manifestations of the disease that share similar clinical presentations and underlying pathophysiology [2], although the natural history may vary widely. In the most recent clinical classification, $\mathrm{PH}$ is classified into five groups. Group 1 is pulmonary arterial hypertension (PAH), which can be hereditary, idiopathic or associated with a number of underlying conditions. Group 2 includes $\mathrm{PH}$ due to left heart disease. Group 3 includes $\mathrm{PH}$ resulting from lung disease and/or hypoxaemia (e.g. interstitial lung disease and chronic obstructive pulmonary disease (COPD)). Group 4 is chronic thromboembolic $\mathrm{PH}$ and group 5 is made up of
$\mathrm{PH}$ with unclear and/or multifactorial mechanisms [2]. Accurate diagnosis and classification of $\mathrm{PH}$ is essential as the prognostic and therapeutic implications are different between the clinical groups [1].

Patients with suspected $\mathrm{PH}$ must be evaluated using a multimodality approach to ensure a correct diagnosis, with a series of investigations ranging from thorough clinical evaluation through noninvasive imaging techniques to $\mathrm{RHC}$, which is considered to be the "gold standard" for the diagnosis of PH [1]. Guidelines recommend that a detailed echocardiographic assessment is performed in all patients with suspected $\mathrm{PH}$ [1]. Transthoracic echocardiography provides a number of measures that can be used to estimate right heart haemodynamics. For example, systolic Ppa calculated from the tricuspid regurgitant velocity (TRV) is used to alert suspicion of $\mathrm{PH}$, although $P$ pa may be underestimated by echocardiography due to an insufficient Doppler envelope or eccentric tricuspid regurgitation jet. In fact, no single

This article has supplementary data available from http://err.ersjournals.com

\section{AFFILIATIONS}

*National Pulmonary Hypertension Service, Hammersmith Hospital, Imperial College Healthcare NHS Trust,

\#Dept of Echocardiography, Imperial College Healthcare NHS Trust,

'Guy's and St Thomas's Hospital, London, and

${ }^{+}$Dept of Cardiology, University Hospital of Wales, Cardiff, UK.

CORRESPONDENCE

L.S. Howard

National Pulmonary Hypertension Service, Dept of Cardiac Sciences Hammersmith Hospital

Du Cane Road

London

W12 OHS

UK

E-mail: I.howard@imperial.ac.uk

Received:

June 082012

Accepted after revision:

July 032012

\section{PROVENANCE}

Publication of this peer-reviewed article was supported by Actelion Pharmaceuticals Ltd, Switzerland (article sponsor, European Respiratory Review issue 125). 
echocardiographic measure is able to diagnose $\mathrm{PH}$ specifically and isolated abnormalities on echocardiography require cautious interpretation. Therefore, it is important that other surrogate echocardiographic measurements of $\mathrm{PH}$ are examined by echocardiography if $\mathrm{PH}$ is suspected. Further assessment of right ventricular function should be considered when one or more of these abnormalities are identified.

We have developed a protocol to describe how various echocardiographic views and measurements may be used during the initial examination and follow-up of PH. These techniques can be used to assess the severity of right ventricle (RV) dysfunction by providing prognostic information and a noninvasive means of following disease progression or response to therapy. The protocol includes only those parameters in regular clinical use, and excludes more specialised parameters such as speckle tracking and three-dimensional imaging, of which there is less experience. The procedure described in the protocol has been used successfully in the UK for the past 3 yrs, is supported by the British Society of Echocardiography and has been approved by the National Pulmonary Hypertension Centres of UK and Ireland. The aim of the protocol is to provide guidance to those undertaking echocardiographic examinations of patients, and also those interpreting the results in a clinical context, including physicians from cardiological and non-cardiological backgrounds.

\section{RV ANATOMY AND PATHOPHYSIOLOGY}

A detailed explanation of the anatomy and pathophysiology of the RV is beyond the scope of this article and has been extensively described elsewhere [3-5]. A number of anatomical and physiological features of the RV and its response to $\mathrm{PH}$ are important when assessing it using echocardiography. Certain anatomical features in particular make it difficult to examine the RV in detail, especially when using two-dimensional (2D) echocardiography. In particular, identifying the borders of the RV can be difficult because of the heavily trabeculated myocardium, while the available image windows can be limited because of the retrosternal position [3].

In contrast to the left ventricle (LV) and the systemic circulation, the pulmonary vascular bed is a low-resistance system in healthy individuals [3]. The RV is more compliant than the LV and adapts better to volume loading than to pressure loading. The LV and RV do not function independently of one another as the mechanics of one ventricle will impact on the other (ventricular interdependence) and this process is mediated by the interventricular septum (IVS) [6]. Ventricular septal geometry and motion are largely determined by the difference in pressure across the ventricular septum. Under normal circumstances, the higher pressures in the LV cavity mean the IVS bows into the lower pressure RV cavity. In patients with $\mathrm{PH}$, pressure in the RV increases causing the septum to flatten in systole as the pressures in both ventricles begin to converge. Eventually, when the RV becomes severely pressure loaded, the septum may even bulge into the LV cavity (fig. 1) [4]. These changes also impact on both LV systolic and diastolic function.

Another change seen in the RV in $\mathrm{PH}$ is ventricular remodelling, which is a result of chronic progressive pressure loading. This is initially in the form of hypertrophy and later as dilation [5]. The remodelling results in progressive contractile impairment, low cardiac output and, eventually, RV failure. Right ventricular
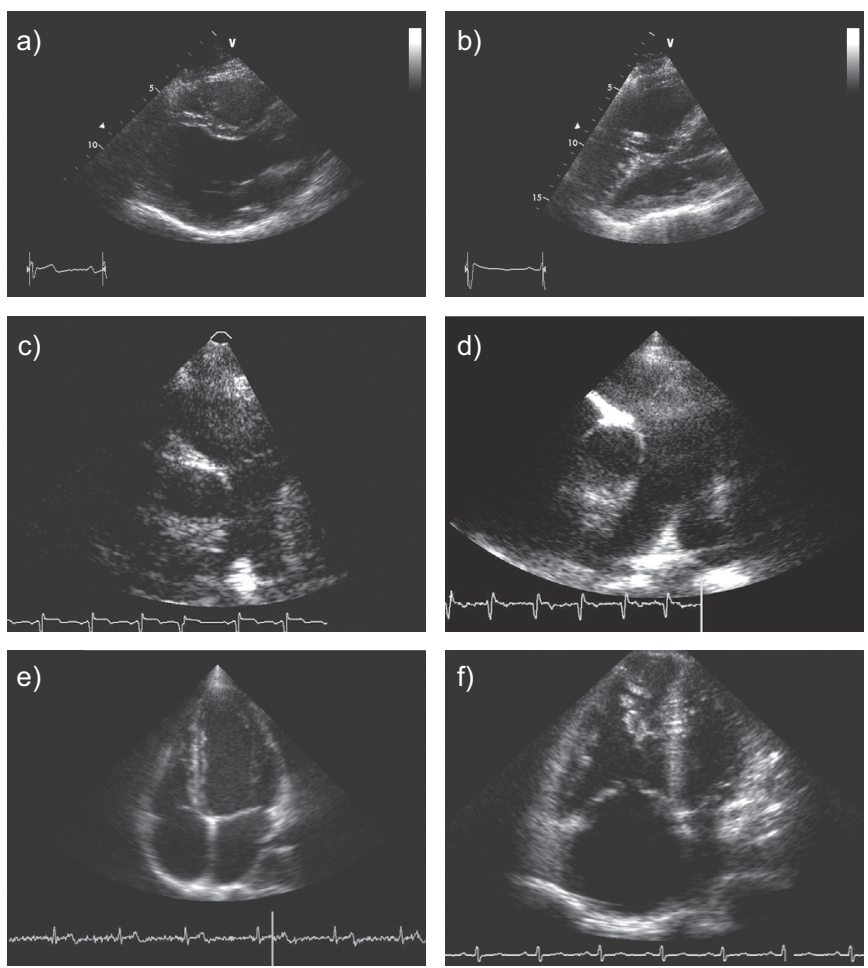

FIGURE 1. a) Normal parasternal long-axis view. Note that the right ventricle is less than one third of the size of the left ventricle. b) Parasternal long-axis view in pulmonary hypertension $(\mathrm{PH})$. Severely dilated right ventricle with hypertrophy of the moderator band and the right ventricular free wall. The left ventricular cavity is small due to chronic right ventricular pressure overload. c) Normal parasternal long-axis view of the right ventricular outflow tract (RVOT) showing the main pulmonary trunk, branches of the pulmonary artery and the pulmonary valve. d) Parasternal long-axis view of the RVOT in $\mathrm{PH}$ showing the dilated pulmonary artery and branches. e) Normal apical four-chamber view. f) Apical four-chamber view in $\mathrm{PH}$ showing marked right ventricular dilation and hypertrophy.

dilation also leads to tricuspid annular dilation and results in significant tricuspid regurgitation [4]. When this is coupled with decreased RV systolic dysfunction and increased afterload it will further reduce stroke volume into the pulmonary circulation and resultant reduced cardiac output. The additional volume loading as a consequence of tricuspid regurgitation will further impair RV diastolic function, increasing RV end-diastolic pressure and displacing the IVS. The progressive deterioration of $\mathrm{RV}$ function is a determinant of exercise capacity, symptoms and prognosis and, therefore, the assessment of RV function is a key component of the assessment of a patient with $\mathrm{PH}$.

\section{RIGHT HEART IMAGING PROTOCOL}

The protocol includes the measurement of a range of parameters assessed from three windows (parasternal, apical and subcostal view), which is first described using Doppler examination and secondly using M-mode examination. The aim of the measures selected is to determine the pressure within, and the capacity and function of, the RV. The technical aspects of the echocardiographic assessment have been described previously [7] and are briefly summarised here. However, the aim of this 
review is to highlight how the findings from the echocardiographic assessment can be interpreted clinically.

\section{Parasternal views}

In normal subjects the anterior RV wall is thin; its mass is less than one sixth and its diameter less than one third of the LV. As previously discussed, chronic pressure loading of the RV in $\mathrm{PH}$ leads to dilation and hypertrophy of the RV; the parasternal views of the RV allow any such changes to be viewed and various parameters to be measured (table 1 ).

In the parasternal long-axis view, the moderator band is usually seen close to the IVS traversing the RV and care should be taken not to include the moderator band in the measurements of the IVS. In significant PH, the LV cavity may be reduced in size in both systole and diastole, with deviation (bowing) of the septum towards the LV (fig. 1a and b).

The long-axis view of the RV inflow tract may also allow for optimal alignment for estimation of TRV when the tricuspid regurgitant jet is eccentric. Abnormalities of the leaflets and subvalvular apparatus should also be assessed to exclude primary valvular pathology. In addition, this view may be used to provide a qualitative estimate of RV systolic function.

The long- and short-axis views of the right ventricular outflow tract (RVOT) are useful for the assessment of the main pulmonary artery, which is dilated in relation to the adjacent aorta in $\mathrm{PH}$, and pulmonary regurgitation (fig. $1 \mathrm{c}$ and $\mathrm{d}$ ). However, we are not aware of any data to suggest that dilation of the pulmonary artery is a sensitive measurement for detection of PH using echocardiography.

As well as demonstrating the atria and RV, the short-axis view of the aorta and left atrium shows the aortic, tricuspid and pulmonary valves simultaneously, making this a useful view for the assessment of structural valvular abnormalities. It can also help to identify an atrial septal defect using a zoom view of the interatrial septum with colour Doppler imaging. RV dilation and hypertrophy, as well as dilation of the pulmonary artery, can also be seen in this view. Finally, the degree of distortion of the LV and IVS arising due to increased pressure in the RV during diastole and systole (LV eccentricity index) can be quantified using a short-axis view at the level of left ventricular papillary muscles. While in a normal situation the LV appears circular in shape, in systole and diastole dilation of the RV and increased RV pressure in severe PH shifts the IVS to the left, giving the LV a characteristic D-shape. Consequently, a RV that is dilated purely due to volume overload will deviate the septum, mainly in diastole, due to raised enddiastolic pressure and, conversely, a pressure-loaded RV will deviate the septum in systole. When pressure overload is severe the septum may even be deviated into the LV cavity itself.

TABLE 1 Parameters affected by pulmonary hypertension (PH) that can be detected by right heart imaging

\begin{tabular}{|c|c|c|c|}
\hline $\begin{array}{l}\text { Long-axis view of LV or short-axis view of } \\
\text { aorta and left atrium }\end{array}$ & LV end-diastolic diameter $\mathrm{cm}$ & 4.2-5.9 in males and 3.9-5.3 in females [8-10] & $\downarrow$ \\
\hline \multirow[t]{3}{*}{ Long-axis view of the RV inflow tract } & Basal diameter of RV cm & $3.7-5.4[11]$ & $\uparrow$ \\
\hline & Tricuspid annulus $\mathrm{cm}$ & $1.3-2.8[11,12]$ & $\uparrow$ \\
\hline & Tricuspid regurgitant velocity $\mathrm{m} \cdot \mathrm{s}^{-1}$ & $<2.6[13]$ & $\uparrow$ \\
\hline \multirow{5}{*}{$\begin{array}{l}\text { Long- and short-axis views of RVOT or short- } \\
\text { axis view of aorta and left atrium }\end{array}$} & Right pulmonary artery $\mathrm{cm}$ & $0.7-1.7[11,14]$ & $\uparrow$ \\
\hline & Left pulmonary artery $\mathrm{cm}$ & $0.6-1.4[8,11]$ & $\uparrow$ \\
\hline & RV outflow acceleration time ms & $>110[8,15,16]$ & $\downarrow$ \\
\hline & Pulmonary regurgitant velocity (beginning of diastole) $\mathrm{m} \cdot \mathrm{s}^{-1}$ & $<1[14,17]$ & $\uparrow$ \\
\hline & Pulmonary regurgitant velocity (end diastole) $\mathrm{m} \cdot \mathrm{s}^{-1}$ & $<1[14,17]$ & $\uparrow$ \\
\hline \multirow[t]{4}{*}{ Apical four-chamber view $w^{\#}$} & Basal diameter of $\mathrm{RV} \mathrm{cm}$ & $2.0-2.8[8,11]$ & $\uparrow$ \\
\hline & RA volume index $\mathrm{mL} \cdot \mathrm{m}^{-2}$ & $\leqslant 34$ in males and $\leqslant 27$ in females $[8,19,20]$ & $\uparrow$ \\
\hline & Tricuspid annulus $\mathrm{cm}$ & $1.3-2.8[8,12]$ & $\uparrow$ \\
\hline & Right ventricular fractional area change $\%$ & $32-60[8,21,22]$ & $\downarrow$ \\
\hline \multirow[t]{5}{*}{ Apical four-chamber view } & Tricuspid regurgitant velocity $\mathrm{m} \cdot \mathrm{s}^{-1}$ & $<2.6[13]$ & $\uparrow$ \\
\hline & Deceleration time-tricuspid inflow ms & $144-244[23,24]$ & $\uparrow$ \\
\hline & RV MPI (Tei index) & $<0.28[8,25,26,27]$ & $\uparrow$ \\
\hline & TAPSE mm & $\geqslant 20[8,28-30]$ & $\downarrow$ \\
\hline & IVRT (TDI RV free wall) ms & $<75[6,8,31,32]$ & $\uparrow$ \\
\hline
\end{tabular}

LV: left ventricle; RV: right ventricle; RVOT: right ventricular outflow tract; RA: right atrium; MPI: myocardial performance index; TAPSE: tricuspid annular plane systolic excursion; IVRT: isovolumic relaxation time; TDI: tissue Doppler imaging; $\downarrow$ : decreased in $\mathrm{PH}$; $\uparrow$ : increased in $\mathrm{PH} .{ }^{*}$ : two-dimensional echocardiograph; " : Doppler echocardiograph. 


\section{Apical four-chamber views}

A number of parameters influenced by the presence of $\mathrm{PH}$ can be assessed in this view (table 1), including RV dilation and hypertrophy, with systolic dysfunction and reduced myocardial tissue velocities being quantifiable by tissue Doppler imaging. In $\mathrm{PH}$, the apex is frequently hypertrophied and akinetic, and careful examination of the apex should be performed to exclude thrombus or an apical mass [7]. Diastolic opening of the tricuspid leaflets, which can also be assessed in this view, becomes less vigorous as $\mathrm{PH}$ progresses. The tricuspid valve leaflet adjacent to the free wall may be either the anterior (most commonly) or posterior leaflet, depending on the exact rotation and angulation of the image plane. Tricuspid leaflets are uniformly echogenic, with normal coaptation in systole. When significant tricuspid regurgitation develops in $\mathrm{PH}$, careful identification of any structural abnormalities is needed to ensure primary tricuspid incompetence is differentiated from secondary incompetence due to the annular dilation.

The interatrial and interventricular septums can be clearly delineated (fig. 1e and f). Drop-out artefact is commonly seen in the region of fossa ovalis and this should not be mistaken for an atrial septal defect. The use of tissue Doppler imaging to the septal and lateral LV walls may help to identify significant LV diastolic dysfunction (table 2).

\section{Subcostal view}

The subcostal view allows the degree of RV dysfunction and the thickness of the RV walls to be estimated. This assessment is particularly useful in patients where parasternal or apical window views are difficult to image, such as those with COPD. It provides the best view for the measurement of RV inferior wall thickness and determines the presence of an atrial septal defect. The diameter of the inferior vena cava measured at rest and during inspiration in this view can be used to provide an estimate of right atrial pressure (Pra).

\section{Doppler examination}

As part of a full echocardiographic assessment, Doppler examination should be performed in the following sequence: 1) colour Doppler in all apical projections; 2) colour Doppler in parasternal projections (long axis/short axis); 3) pulsed-wave Doppler for transmitral velocities; 4) pulsed-wave Doppler for LV outflow tract; 5) pulsed-wave Doppler for the tricuspid inflow; 6) pulsed-wave Doppler for the RVOT; 7) continuous-wave Doppler across the LV outflow aortic valve; 8) continuous-wave Doppler across the tricuspid valve (for tricuspid regurgitation); 9) continuous-wave Doppler across the pulmonary valve (for pulmonary regurgitation); and 10) tissue Doppler imaging (TDI) of the RV free wall.

\section{QUALITATIVE ASSESSMENT OF THE RV}

Dilation of the RV can be assessed in the parasternal long-axis, short-axis and the apical four-chamber views. When RV afterload becomes chronically elevated, the RV walls become hypertrophied. RV hypertrophy is defined by a free wall thickness of $>5 \mathrm{~mm}$ on the apical four-chamber view. One of the first anatomical elements to become hypertrophied is the moderator band. In normal subjects this is thin and difficult to see, but in patients with $\mathrm{PH}$ it is clearly visible. In contrast to other conditions affecting the RV (e.g. RV infarction or arrhythmogenic RV cardiomyopathy) where there will be regional wall motion abnormalities, contractility assessment in PH shows global RV impairment. On the basis of these three parameters (dilation, hypertrophy and contractility), an experienced echocardiographer will be able to make a good qualitative assessment of RV function and will be able to grade it as mild, moderate or severe impairment. In patients with $\mathrm{PAH}$, dilation of the RV has been shown to be linked to adverse clinical outcome (increase of $>0.25$ in absolute value of RV sphericity index; i.e. ratio of the short axis at the midventricular level of the RV divided by the RV long axis in enddiastole [36]) and mortality (RV diameter $>36.5 \mathrm{~mm}$ ) [37].

\section{PRESSURE MEASUREMENTS IN PH}

Pra can be estimated by measurement of the diameter of the inferior vena cava at end-expiration and during sharp inspiration using M-mode in the subcostal view (fig. 2d). High Pra is associated with dilation of the vena cava (normal range 1.5$2.5 \mathrm{~cm}$ ) and/or failure of the segment adjacent to the right atrium to collapse by at least $50 \%$ with inspiration (table 3 ). Inferior vena cava diameter $\geqslant 20 \mathrm{~mm}$ with respiratory variation of diameter $<50 \%$ has been shown to be significantly associated with mortality in patients with PAH [38].

TRV reflects the difference in pressure between the RV and the right atrium and can be measured from continuous wave Doppler of the tricuspid regurgitant jet from the apical fourchamber view, or from the parasternal RV inflow view if the regurgitant jet is eccentric (fig. 2a). When pulmonary stenosis is absent, the RV systolic pressure (RVSP) is assumed to be equivalent to the systolic $P$ pa and can be calculated from the TRV using the Bernoulli equation, using estimated Pra from inferior vena cava diameter (table 3) [7].

$$
\text { systolic } P \text { pa }=\mathrm{RVSP}=4(\mathrm{TRV} \text { end })^{2}+P \text { ra }
$$

TABLE 2 Effect of pulmonary hypertension (PH) on measurements from tissue Doppler imaging

\begin{tabular}{|c|c|c|c|c|c|c|c|}
\hline Measurements & [Ref.] & Normal S' wave $\mathrm{cm} \cdot \mathrm{s}^{-1}$ & $\mathrm{PH}$ & Normal E' $\mathrm{cm} \cdot \mathrm{s}^{-1}$ & $\mathrm{PH}$ & Normal A' $\mathrm{cm} \cdot \mathrm{s}^{-1}$ & $\mathrm{PH}$ \\
\hline RV free (lateral) wall & [33-35] & $12-20$ & $\downarrow$ & $10.2-16.2$ & $\downarrow$ & $6.2-10.9$ & $\downarrow$ \\
\hline LV septal wall & [34] & $8.1-10.9$ & $\leftrightarrow$ & $9.8-16.0$ & $\leftrightarrow$ & $9.0-13.2$ & $\leftrightarrow$ \\
\hline
\end{tabular}

S': systolic wave; A': late (atrial systole) myocardial diastolic wave; E': early myocardial diastolic wave; RV: right ventricle; LV: left ventricle; $\downarrow$ : decreased in PH; $\leftrightarrow$ : no change. 

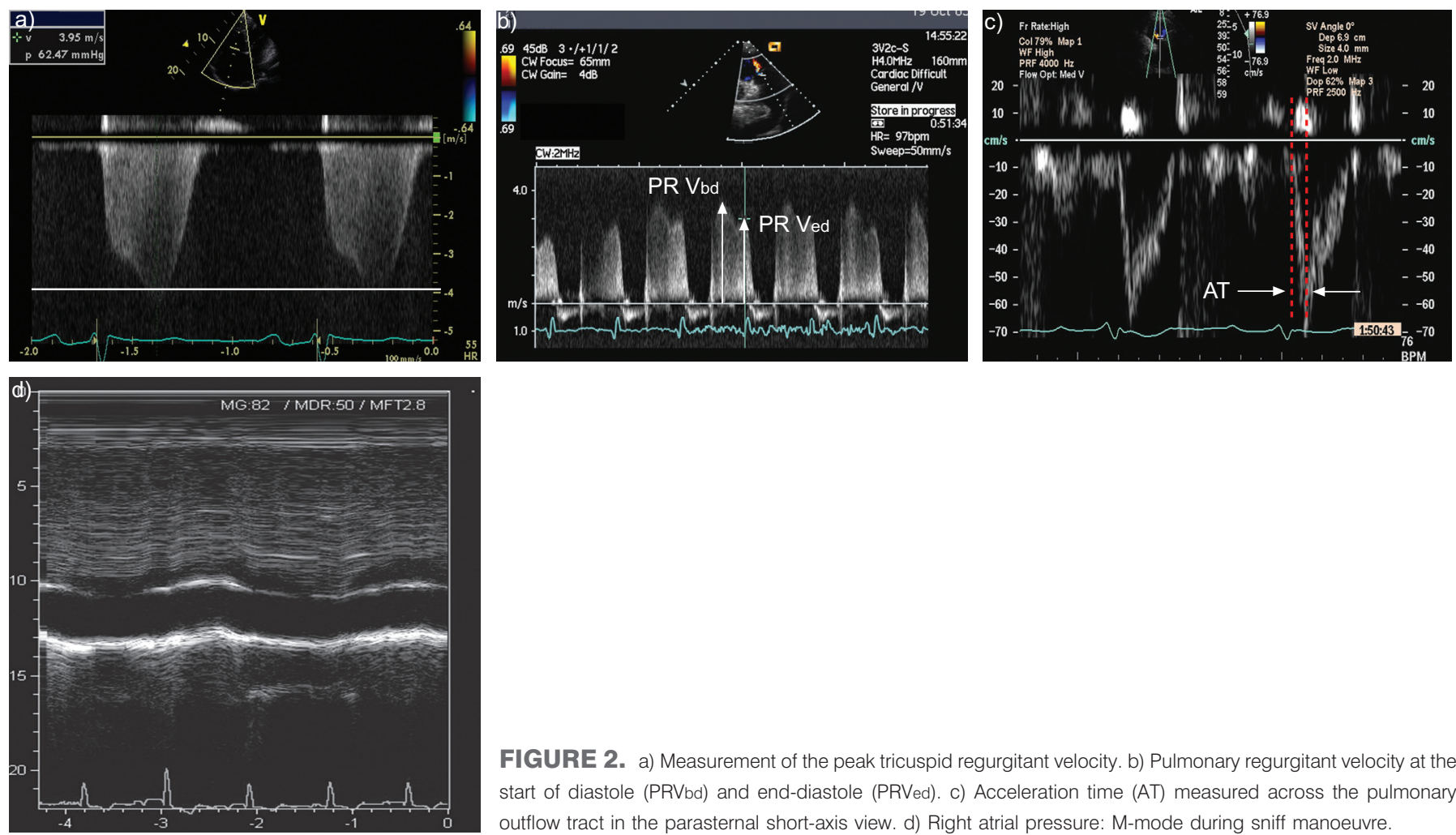

FIGURE 2. a) Measurement of the peak tricuspid regurgitant velocity. b) Pulmonary regurgitant velocity at the start of diastole (PRVbd) and end-diastole (PRVed). c) Acceleration time (AT) measured across the pulmonary outflow tract in the parasternal short-axis view. d) Right atrial pressure: M-mode during sniff manoeuvre.

where TRVend is peak TRV at end diastole. The ability to estimate systolic $P$ pa represents a considerable strength of echocardiography in its role as a diagnostic tool, but with this comes limitations and there is a danger that too much weight is placed on this single measurement. FISHER et al. [39] have shown that, despite good correlation between measurements, almost $50 \%$ of patients had an estimated systolic $P$ pa on echocardiography that was $\geqslant 10 \mathrm{mmHg}$ different from the value measured at cardiac catheterisation. Underestimation of systolic $P$ pa has led to frequent misclassification of $\mathrm{PH}$ severity or even failure to identify $\mathrm{PH}$ [39]. The most common causes of inaccurate estimation of systolic $P$ pa were an incomplete Doppler envelope resulting in underestimation of pressure or an overestimate of $P$ ra from inferior vena cava diameter and collapsibility. With severe freeflow tricuspid regurgitation, the Bernoulli equation cannot be

\begin{tabular}{|c|c|c|}
\hline $\begin{array}{l}\text { Inferior vena } \\
\text { cava diameter }\end{array}$ & $\begin{array}{l}\text { Change with } \\
\text { respiration }\end{array}$ & $\begin{array}{l}\text { Estimated right atrial } \\
\text { pressure } \mathrm{mmHg}\end{array}$ \\
\hline Small $<1.5 \mathrm{~cm}$ & Collapse & 0 \\
\hline Normal $1.5-2.5 \mathrm{~cm}$ & Decrease by $>50 \%$ & 5 \\
\hline Normal & Decrease by $<50 \%$ & 10 \\
\hline Dilated $>2.5 \mathrm{~cm}$ & Decrease by $<50 \%$ & 15 \\
\hline $\begin{array}{l}\text { Dilated with dilated } \\
\text { hepatic veins }\end{array}$ & No change & 20 \\
\hline
\end{tabular}

applied and TRV will underestimate the trans-tricuspid pressure difference. In itself, the severity of the tricuspid regurgitation is predictive of survival regardless of the systolic $P$ pa and irrespective of the underlying disease $[37,40]$. Tricuspid regurgitation may be assessed in a number of ways, including assessment of the structure of the valve, jet area and density or measurement using continuous wave Doppler [41]. It should be emphasised that the severity of the volume of tricuspid regurgitation is distinct from the velocity. Thus, Doppler-derived estimates of RVSP/systolic $P$ pa must only be considered to be estimates [1, 39, 42] and cannot be used to diagnose PH. For this reason, it is often valuable to consider other surrogates for $P$ pa.

Mean $P$ pa and pulmonary end-diastolic pressure are not routinely used in the diagnosis or follow-up of $\mathrm{PH}$, but may be useful when TRV cannot be used or is unreliable. In 2D-echocardiography, the pulmonary valve is best imaged from the parasternal short-axis at the level of the aortic valve (fig. 1c and 1d). Pulmonary valvular motion can be determined with M-mode echocardiography and may show mid-diastolic closure. Mean Ppa and pulmonary enddiastolic pressure can be derived from the TRV at the beginning (PRVbd) and end (PRVed) of diastole, respectively (fig. 2b).

$$
\begin{gathered}
\text { pulmonary end-diastolic pressure }=4(\text { PRVed })^{2}+\text { Pra } \\
\mathrm{mP} P \text { pa }=4(\text { PRVbd })^{2}
\end{gathered}
$$

RVOT acceleration time has been superseded by TRV in clinical practice, although it may be used when the tricuspid velocity cannot be measured, particularly at diagnosis. A value $<105 \mathrm{~ms}$ is suggestive of $\mathrm{PH}$. By positioning the sample volume at the centre of the pulmonary artery (ideally at the annulus) in the short-axis view, acceleration time can be measured in the 
parasternal short-axis view (fig. 2c). Unlike most other indices of function, which are unaffected by heart rate, RVOT acceleration time should be corrected when the heart rate is $>100 \mathrm{bpm}$ or $<70 \mathrm{bpm}$. This is achieved by multiplying the measurement by 75 and dividing by heart rate. Notching of the flow velocity envelope may also be seen in PH. A notch seen in mid-systole is associated with severe $\mathrm{PH}$ and $\mathrm{RV}$ dysfunction [43]. Normal pressures and those in PH obtained by echocardiography are shown in table 4 .

\section{CARDIAC CHAMBER CAVITY MEASUREMENTS IN PH}

Right atrial volume index is calculated from the apical fourchamber view or from the subcostal view, and is measured at maximum atrial volume at end-systole. The single plane arealength method is used and right atrium volume is measured using the area and the long-axis length of the atrium (fig. 3a and b) $[8,19,20]$.

\section{right atrium volume index $=\left(0.85 \mathrm{~A}^{2} / \mathrm{L}\right) / \mathrm{BSA}$}

where $\mathrm{A}$ is the atrium area in any view $\left(\mathrm{cm}^{2}\right), \mathrm{L}$ is the long-axis atrium length $(\mathrm{cm})$ and BSA is body surface area.

In a recent three-dimensional echocardiography study, while both right atrium and RV remodelling were found to be significantly associated with adverse clinical outcome, sensitivity and specificity were highest for right atrium remodelling [36]. An increase of right atrium sphericity index (ratio of the short axis of the right atrium, parallel to the tricuspid annulus, mid-cavity, divided by the long axis, perpendicular to the tricuspid annulus, in end systole) $>0.24$ predicted clinical deterioration with a sensitivity of $96 \%$ and a specificity of $90 \%$ [36].
RV fractional area change is calculated from end-diastolic area and end-systolic area, measured from the apical four-chamber view. It is a simple method for the assessment of RV systolic function and has been shown to correlate with prognosis and response to treatment in $\mathrm{PH}$ [48], and with survival [37]. However, it is limited by difficulties in endocardial definition.

$$
\text { RV fractional area change }(\%)=
$$

(end-diastolic area - end-systolic area)/end-diastolic area

LV eccentricity index is the ratio of the minor axis of the LV parallel to the septum (D2) divided by the minor axis perpendicular to the septum (D1) and is measured in the parasternal short-axis view at the level of the LV papillary muscles (fig. 3c and d) in both end diastole and end systole. In a purely pressure-loaded RV, flattening of the IVS occurs in end systole, resulting in an increased end-systolic LV eccentricity index. Conversely, the eccentricity index will be increased in end diastole in pure volume loading [8, 44, 45]. LV diastolic eccentricity index in diastole $\geqslant 1.7$ has been shown to be prognostic of mortality in patients with idiopathic PAH who also have reduced RV function (tricuspid annular plane systolic exertion (TAPSE) $\leqslant 15 \mathrm{~mm}$ ) [37]. Normal cavity measurements and those in $\mathrm{PH}$ as obtained by echocardiography are shown in table 4.

\section{MEASURES OF FUNCTION IN PH}

Myocardial performance index of the RV, also known as the Tei index [49], is made up of a combination of systolic and diastolic measurements. It has a number of advantages as a functional measure, including: 1) being relatively unaffected

\section{TABLE 4 Right ventricular pressure, cavity and functional measurements that may indicate pulmonary hypertension}

\begin{tabular}{|c|c|c|c|c|}
\hline Parameter & [Ref.] & Normal & Intermediate & Abnormal \\
\hline Pra $\mathrm{mmHg}$ & {$[8]$} & $<5$ & $5-10$ & $>10$ \\
\hline \multirow[t]{2}{*}{ TRV $\mathbf{m} \cdot \mathbf{s}^{-1}$} & {$[13]$} & $<2.6$ & & $\geqslant 2.6$ \\
\hline & & & & $\geqslant 2.9$ in patients $>60 \mathrm{yrs}$ \\
\hline RVSP $\mathrm{mmHg}$ & {$[13]$} & $<37$ & & $\geqslant 37$ \\
\hline RVOT acceleration time ms & {$[8,15,16]$} & $>110$ & $105-110$ & $<105$ \\
\hline \multirow[t]{2}{*}{$\mathrm{RA}$ volume index $\mathrm{mL} \cdot \mathrm{m}^{-2}$} & {$[8]$} & $\leqslant 34$ in males and & & $>34$ in males \\
\hline & & $\leqslant 27$ in females & & $>27$ in females \\
\hline RV fractional area change \% & {$[8,21,22]$} & $32-60$ & & $\leqslant 32$ \\
\hline LV eccentricity index & {$[8,44,45]$} & 1 & & $>1$ at end-diastole indicates \\
\hline & & & & volume loading of the RV \\
\hline IVRT s & {$[6,8,31,32]$} & $<75$ & & $\geqslant 75$ \\
\hline TAPSE ms & {$[8,28-30]$} & $\geqslant 20$ & $16-20$ & $<16$ \\
\hline Estimated PVR Wood units & {$[46,47]$} & $<1$ & $1-3$ & $>3$ \\
\hline
\end{tabular}

Pra: right atrial pressure; TRV: tricuspid regurgitant velocity; RVSP: right ventricular systolic pressure; RVOT: right ventricular outflow tract; RA: right atrium; RV: right ventricle; LV: left ventricle; MPI: myocardial performance index; S' wave: systolic wave; IVRT: isovolumic relaxation time; TAPSE: tricuspid annular plane systolic exertion; PVR: pulmonary vascular resistance. 

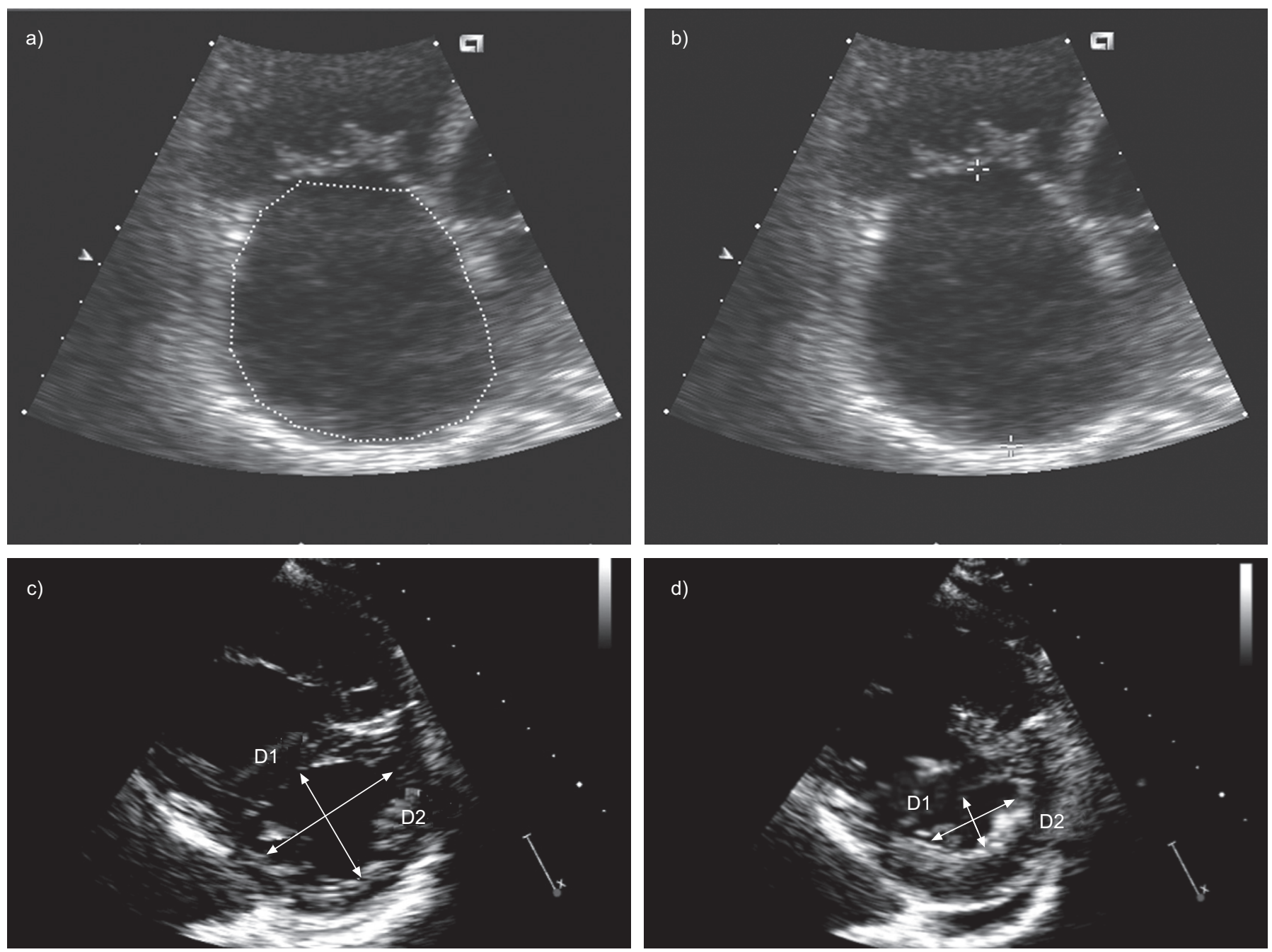

FIGURE 3. a) Measurement of the right atrial a) area and b) long axis for calculation of right atrial volume. c) Measurement of the left ventricular eccentricity index in c) end-diastole and d) end-systole. DI: minor axis perpendicular to the septum; D2: minor axis of the left ventricle parallel to the septum.

by heart rate, loading conditions or the presence and severity of tricuspid regurgitation; 2) having good reproducibility; 3) being quick to calculate; 4) not relying on the use of geometric models; and 5) being able to be applied even in the presence of a difficult acoustic window.

The Tei index can be measured either from colour Doppler imaging (apical four-chamber view for the tricuspid inflow pattern and the parasternal short-axis RVOT view for the determination of ejection time) or tissue Doppler imaging (fig. 4a). The Tei index should be indexed for heart rate, as described previously for RVOT acceleration time. In patients with idiopathic PAH the index correlates with symptoms and values $>0.88$ predict poor survival [25].

Systolic $\left(S^{\prime}\right)$ wave velocity is a measure of myocardial contraction and may be determined from the average of three TDI signals from different cardiac cycles (fig. 4a). Like TAPSE, S' wave velocity is load dependent and may be pseudonormal under conditions of increased volume loading (this is discussed in more detail below). Unlike most indices of function, $\mathrm{S}^{\prime}$ wave velocity requires correction when heart rate is $>100 \mathrm{bpm}$ or falls to $<70 \mathrm{bpm}$. Therefore, $\mathrm{S}^{\prime}$ wave velocity should be indexed for heart rate, as described previously for RVOT acceleration time. Normal values and those in $\mathrm{PH}$ obtained by echocardiography are shown in table 4.

Isovolumic relaxation time (IVRT) of the RV is defined as the time from pulmonary valve closure to tricuspid valve opening and can be measured either by pulsed-wave Doppler from tricuspid inflow or with tissue Doppler imaging of the RV free wall. Prolongation of IVRT indicates poor myocardial relaxation, which is highly suggestive of $\mathrm{PH}$, although it does not provide prognostic value (fig. 4a). IVRT should be indexed for heart rate as described previously for RVOT acceleration time.

TAPSE is the reflection of the movement of base to apex shortening of the RV in systole and can be derived from the four-chamber view (fig. 4b). When measuring TAPSE, it is important to ensure that the entire RV is included in the view, in particular that there is no dropout in the endocardial outline along the IVS and RV free wall. Maximal TAPSE is defined by the total excursion of the tricuspid annulus from its highest position after atrial ascent to the peak descent during ventricular systole [29]. A TAPSE of $<15 \mathrm{~mm}$ is associated with a significantly higher risk of mortality compared with a TAPSE 

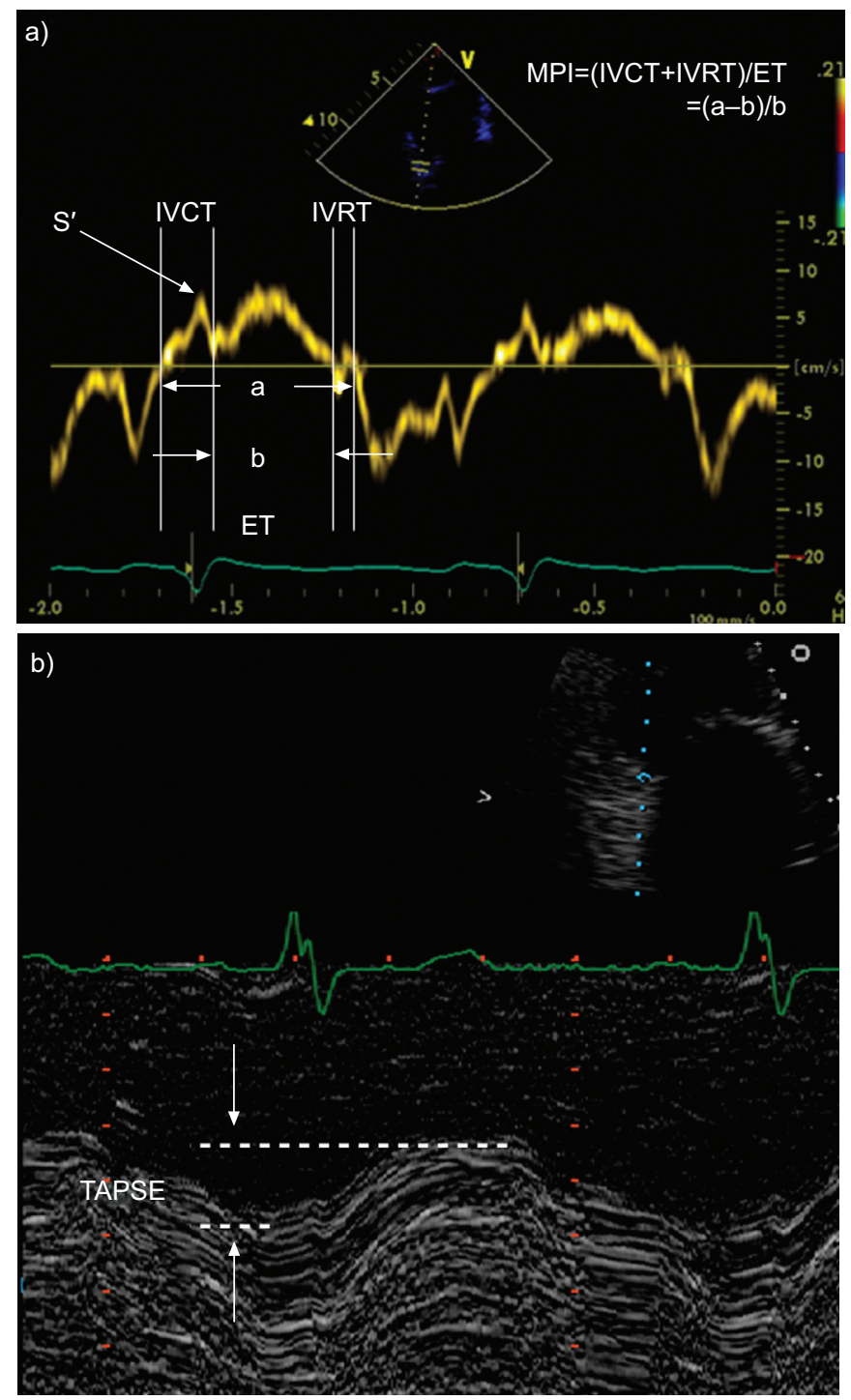

FIGURE 4. a) Measurement of myocardial performance index (MPI) using tissue Doppler imaging. S': systolic wave; IVCT: isovolumic contraction time; IVRT: isovolumic relaxation time. b) Measurement of tricuspid annular plane systolic excursion (TAPSE); ET: ejections time.

of $>15 \mathrm{~mm}$ [37] and TAPSE is recommended by treatment guidelines as a prognostic indicator in $\mathrm{PAH}$ for the assessment of disease severity and response to therapy [1]. A significant limitation of TAPSE is that it is highly load dependent, such that it may become pseudonormalised in the presence of significant volume loading, e.g. left-to-right shunting or severe functional tricuspid regurgitation [37]. Given that so much weight is placed on this particular measure of function in the treatment guidelines, it is important that this caveat is taken into account when interpreting TAPSE.

Stroke volume, cardiac output and pulmonary vascular resistance can be calculated from echocardiographic measurements. These parameters correlate with right and left heart function and the underlying pulmonary vascular resistance, but are not considered to be mandatory measures in PH. Normal values and those in $\mathrm{PH}$ obtained by echocardiography are shown in table 4 .
Is PH suspected?

$\mathrm{TRV} \geq 2.6 \mathrm{~m} \cdot \mathrm{s}^{-1}[13]^{\#}$

End-diastolic PRV $>1.0 \mathrm{~m} \cdot \mathrm{s}^{-1}[14,17]$

$R V \geq 1 / 2$ LV from PLAX [11]

RVOT AT <105 ms $[8,15,16]$

TAPSE $<20 \mathrm{~mm}[8,28-30,33,53,54]$

RV IVRT $>75 \mathrm{~ms}[8,31,32]$

IVC $>20 \mathrm{~mm}$ and $<50 \%$ inspiratory collapse [8]

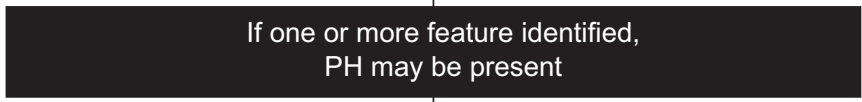

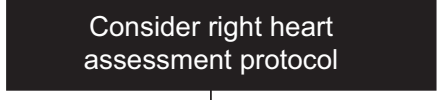

RA volume $[8,20]$

RV myocardial

performance index [8, 25-27]

Tissue Doppler index of $\mathrm{RV}$ free wall [7, 8, 31, 32, 34]

Eccentricity index (end-systolic and diastolic) $[8,43,44]$

Cardiac output $[46,47]$

Pulmonary vascular resistance $[46,47]$

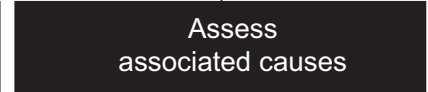

Congenital heart disease (in particular, exclude pulmonary stenosis)

Valvular heart disease (in particular, mitral valve)

LV systolic/diastolic dysfunction
FIGURE 5. An algorithm for investigating pulmonary hypertension $(\mathrm{PH})$ using echocardiography. TRV: tricuspid regurgitant velocity; PRV: pulmonary regurgitant velocity; RV: right ventricle; LV left ventricle; PLAX: parasternal long axis; RVOT: right ventricular outflow tract; AT: acceleration time; TAPSE: tricuspid annular plane systolic excursion; IVRT: isovolumic relaxation time; IVC: inferior vena cava; RA: right atrium. ${ }^{*}$ : in patients $>60 \mathrm{yrs}$ of age, a TRV $\geqslant 2.9 \mathrm{~m} \cdot \mathrm{s}^{-1}$ is used as a cut-off value [13]

\section{CAUSES OF PH}

Once PH has been suspected, further evaluation should be undertaken to establish any potential underlying cause. This includes assessment for congenital heart disease, valvular heart disease and left heart disease. These assessments are out of the scope of this protocol, but as the most frequent cause of $\mathrm{PH}$ will relate to impaired LV function, it is imperative to highlight that a detailed assessment of diastolic LV function is undertaken. In our experience this is something that is often overlooked. This should include measurement of left atrial diameter and volume (indexed for body surface area), LV hypertrophy, transmitral inflow patterns, tissue Doppler imaging of the basal lateral wall of the LV and, in some cases, pulmonary venous flow. Of note, type I diastolic dysfunction of the LV is common in pre-capillary $\mathrm{PH}$ and should not be considered as indicative of pulmonary venous hypertension as the cause of $\mathrm{PH}$. This has been reviewed comprehensively 
elsewhere, with a proposed scoring system for differentiating between pulmonary venous hypertension and PAH [50].

\section{NEW TECHNIQUES}

We have presented a core clinical dataset for $\mathrm{PH}$ echocardiography. Outside this dataset other techniques can be applied, but these do not form part of routine investigation either as a result of complexity or because of lack of clinical validation. Exercise echocardiography has been proposed for early detection of subclinical $\mathrm{PH}$, e.g. in relatives of patients with idiopathic $\mathrm{PAH}$ [51], but as yet no clear clinical cut-off values have been accepted. Strain and strain rate examine the deformation and rate of deformation, respectively, of the myocardial segment and, in PH, RV systolic strain and strain rate has been shown to predict morbidity and mortality [52], but at present is still considered outside the standard echocardiography protocol.

\section{ECHOCARDIOGRAPHY REPORTING}

All echocardiographic reports should include measurements, descriptions of all valves, the proximal great arteries, the heart chambers, the pericardium and Doppler findings. Where $\mathrm{PH}$ is the reason for the referral, the emphasis should be put on pulmonary pressures, right heart chamber size and function and any finding that may indicate a secondary cause (e.g. LV dysfunction, left heart valve disease or congenital heart disease). Where $\mathrm{PH}$ is confirmed, the report should also include the various parameters that provide prognostic information (e.g. right atrial volume index, inferior vena cava diameter, eccentricity index, presence of pericardial effusion and TAPSE). It is important that the reporter does not judge severity of PAH on the basis of estimated Ppa. Instead, conclusions should be drawn on the severity of RV impairment. An example report is shown in figure S1 (online supplementary data).

These criteria should be taken as guides, not rules, and have been selected by consensus: it is important not to place too much emphasis on a single value, or consider them as precise thresholds for the diagnosis of $\mathrm{PH}$. It may be necessary to undertake catheterisation when clinical suspicion is high, since echocardiography may sometimes be normal even in the presence of $\mathrm{PH}$. For example, in patients with a high pre-test probability of $\mathrm{PH}$ with unexplained breathlessness (such as those with scleroderma), as pressure can only be estimated by echocardiography, the final diagnosis of $\mathrm{PH}$ can only be made by cardiac catheterisation.

\section{CONCLUSIONS}

Detailed echocardiographic assessment of patients with $\mathrm{PH}$ allows useful diagnostic information to be collected. It can also be used to assess severity of right ventricular dysfunction, providing prognostic information and a noninvasive means of following disease progression or response to therapy. A suggested algorithm for investigating $\mathrm{PH}$ using transthoracic echocardiography is shown in figure 5.

\section{STATEMENT OF INTEREST}

P. Nihoyannopoulos has received research and educational grants from General Electric and from BRACCO Pharmaceuticals for organising educational meetings. He has also received fees (less than $£ 1,000$ ) for consulting from General Electric.

\section{ACKNOWLEDGEMENTS}

We thank A. Kenny for her comments on the standard operating procedure. Medical writing support was provided by L. Quine (Elements Communications Ltd, Westerham, UK) and funded by Actelion (Chiswick, UK).

\section{REFERENCES}

1 Galiè N, Hoeper MM, Humbert M, et al. Guidelines for the diagnosis and treatment of pulmonary hypertension. Eur Respir $J$ 2009; 34: 1219-1263.

2 Simonneau G, Robbins IM, Beghetti M, et al. Updated clinical classification of pulmonary hypertension. J Am Coll Cardiol 2009; 54: Suppl. 1, S43-S54.

3 Haddad F, Couture P, Tousignant C, et al. The right ventricle in cardiac surgery, a perioperative perspective: I. Anatomy, physiology, and assessment. Anesth Analg 2009; 108: 407-421.

4 Haddad F, Couture $\mathrm{P}$, Tousignant $\mathrm{C}$, et al. The right ventricle in cardiac surgery, a perioperative perspective: II. Pathophysiology, clinical importance, and management. Anesth Analg 2009; 108: 422-433.

5 Bogaard HJ, Abe K, Vonk Noordegraaf A, et al. The right ventricle under pressure: cellular and molecular mechanisms of right-heart failure in pulmonary hypertension. Chest 2009; 135: 794-804.

6 Santamore WP, Dell'Italia LJ. Ventricular interdependence: significant left ventricular contributions to right ventricular systolic function. Prog Cardiovasc Dis 1998; 40: 289-308.

7 Grapsa J, Dawson D, Nihoyannopoulos P. Assessment of right ventricular structure and function in pulmonary hypertension. J Cardiovasc Ultrasound 2011; 19: 115-125.

8 Lang RM, Bierig M, Devereux RB, et al. Recommendations for chamber quantification: a report from the American Society of Echocardiography's Guidelines and Standards Committee and the Chamber Quantification Writing Group, developed in conjunction with the European Association of Echocardiography, a branch of the European Society of Cardiology. J Am Soc Echocardiogr 2005; 18: 1440-1463.

9 Ilercil A, O'Grady MJ, Roman MJ, et al. Reference values for echocardiographic measurements in urban and rural populations of differing ethnicity: the Strong Heart Study. J Am Soc Echocardiogr 2001; 14: 601-611.

10 Schiller NB, Shah PM, Crawford M, et al. Recommendations for quantitation of the left ventricle by two-dimensional echocardiography. American Society of Echocardiography Committee on Standards, Subcommittee on Quantitation of Two-Dimensional Echocardiograms. J Am Soc Echocardiogr 1989; 2: 358-367.

11 Foale R, Nihoyannopoulos P, McKenna W, et al. Echocardiographic measurement of the normal adult right ventricle. Br Heart J 1986; 56: 33-44.

12 Kitzman DW, Scholz DG, Hagen PT, et al. Age-related changes in normal human hearts during the first 10 decades of life. Part II (Maturity): a quantitative anatomic study of 765 specimens from subjects 20 to 99 years old. Mayo Clin Proc 1988; 63: 137-146.

13 McQuillan BM, Picard MH, Leavitt M, et al. Clinical correlates and reference intervals for pulmonary artery systolic pressure among echocardiographically normal subjects. Circulation 2001; 104: 2797-2802.

14 Yoshida K, Yoshikawa J, Shakudo M, et al. Color Doppler evaluation of valvular regurgitation in normal subjects. Circulation 1988; 78: 840-847.

15 Kitabatake $\mathrm{A}$, Inoue $\mathrm{M}$, Asao $\mathrm{M}$, et al. Noninvasive evaluation of pulmonary hypertension by a pulsed Doppler technique. Circulation 1983; 68: 302-309.

16 Maeba H, Nakatani S, Sugawara M, et al. Different time course of changes in tricuspid regurgitant pressure gradient and pulmonary artery flow acceleration after pulmonary thromboendarterectomy: 
implications for discordant recovery of pulmonary artery pressure and compliance. Circ J 2007; 71: 1771-1775.

17 Vahanian A, Baumgartner H, Bax J, et al. Guidelines on the management of valvular heart disease: The Task Force on the Management of Valvular Heart Disease of the European Society of Cardiology. Eur Heart J 2007; 28: 230-268.

18 Triulizi M, Gillam LD, Gentile F, et al. Normal adult cross-sectional echocardiographic values: linear dimensions and chamber areas echocardiography. Echocardiography 1984; 1: 403-426.

19 Schnittger I, Gordon EP, Fitzgerald PJ, et al. Standardized intracardiac measurements of two-dimensional echocardiography. J Am Coll Cardiol 1983; 2: 934-938.

20 Wang Y, Gutman JM, Heilbron D, et al. Atrial volume in a normal adult population by two-dimensional echocardiography. Chest 1984; 86: 595-601.

21 Anavekar NS, Skali H, Bourgoun $M$, et al. Usefulness of right ventricular fractional area change to predict death, heart failure, and stroke following myocardial infarction (from the VALIANT ECHO Study). Am J Cardiol 2008; 101: 607-612.

22 Wang J, Prakasa K, Bomma C, et al. Comparison of novel echocardiographic parameters of right ventricular function with ejection fraction by cardiac magnetic resonance. I Am Soc Echocardiogr 2007; 29: 1058-1064.

23 Appleton CP, Firstenberg MS, Garcia MJ, et al. The echo-Doppler evaluation of left ventricular diastolic function. A current perspective. Cardiol Clin 2000; 18: 513-546.

24 Hatle L, Angelsen B. Doppler Ultrasound in Cardiology. Physical Principles and Clinical Applications. 2nd Edn. Philadelphia, Lea \& Febiger, 1985.

25 Tei C, Dujardin KS, Hodge DO, et al. Doppler echocardiographic index for assessment of global right ventricular function. J Am Soc Echocardiogr 1996; 9: 838-847.

26 Dujardin KS, Tei C, Yeo TC, et al. Prognostic value of a Doppler index combining systolic and diastolic performance in idiopathicdilated cardiomyopathy. Am J Cardiol 1998; 82: 1071-1076.

27 Yeo TC, Dujardin KS, Tei C, et al. Value of a Doppler-derived index combining systolic and diastolic time intervals in predicting outcome in primary pulmonary hypertension. Am J Cardiol 1998; 81: 1157-1161.

28 Forfia PR, Fisher MR, Mathai SC, et al. Tricuspid annular displacement predicts survival in pulmonary hypertension. Am J Respir Crit Care Med 2006; 174: 1034-1041.

29 Kaul S, Tei C, Hopkins JM, et al. Assessment of right ventricular function using two-dimensional echocardiography. Am Heart J 1984; 107: 526-531.

30 Lee CY, Chang SM, Hsiao SH, et al. Right heart function and scleroderma: insights from tricuspid annular plane systolic excursion. Echocardiography 2007; 24: 118-125.

31 Abbas A, Lester S, Moreno FC, et al. Noninvasive assessment of right atrial pressure using Doppler tissue imaging. I Am Soc Echocardiogr 2004; 17: 1155-1160.

32 Stein PD, Sabbah HN, Anbe DT, et al. Performance of the failing and nonfailing right ventricle of patients with pulmonary hypertension. Am J Cardiol 1979; 44: 1050-1055.

33 Olson JM, Samad BA, Alam M. Prognostic value of pulse-wave tissue Doppler parameters in patients with systolic heart failure. Am J Cardiol 2008; 102: 722-725.

34 Alam M, Wardell J, Andersson E, et al. Characteristics of mitral and tricuspid annular velocities determined by pulsed wave Doppler tissue imaging in healthy subjects. J Am Soc Echocardiogr 1999; 12: 618-628.

35 Meluzin J, Spinarova L, Bakala J, et al. Pulsed Doppler tissue imaging of the velocity of tricuspid annular systolic motion: a new rapid and non-invasive method of evaluating right ventricular systolic function. Eur Heart J 2001; 22: 340-348.
36 Grapsa J, Gibbs JS, Cabrita IZ, et al. The association of clinical outcome with right atrial and ventricular remodelling in patients with pulmonary arterial hypertension: study with real-time threedimensional echocardiography. Eur Heart J Cardiovasc Imaging 2012; [Epub ahead of print DOI: 10.1093/ehjci/jes003].

37 Ghio S, Klersy C, Magrini G, et al. Prognostic relevance of the echocardiographic assessment of right ventricular function in patients with idiopathic pulmonary arterial hypertension. Int $J$ Cardiol 2010; 140: 272-278.

38 Brierre G, Blot-Souletie N, Degano B, et al. New echocardiographic prognostic factors for mortality in pulmonary arterial hypertension. Eur J Echocardiogr 2010; 11: 516-522.

39 Fisher MR, Forfia PR, Chamera E, et al. Accuracy of Doppler echocardiography in the hemodynamic assessment of pulmonary hypertension. Am J Respir Crit Care Med 2009; 179: 615-621.

40 Nath J, Foster E, Heidenreich PA. Impact of tricuspid regurgitation on long-term survival. J Am Coll Cardiol 2004; 43: 405-409.

41 Zoghbi WA, Enriquez-Sarano M, Foster E, et al. Recommendations for evaluation of the severity of native valvular regurgitation with two-dimensional and Doppler echocardiography. J Am Soc Echocardiogr 2003; 16: 777-802.

42 Mukerjee D, St George D, Knight C, et al. Echocardiography and pulmonary function as screening tests for pulmonary arterial hypertension in systemic sclerosis. Rheumatology (Oxford) 2004; 43: 461-466.

43 Arkles JS, Opotowsky AR, Ojeda J, et al. Shape of the right ventricular Doppler envelope predicts hemodynamics and right heart function in pulmonary hypertension. Am J Respir Crit Care Med 2011; 183: 268-276.

44 Feneley M, Gavaghan T. Paradoxical and pseudoparadoxical interventricular septal motion in patients with right ventricular volume overload. Circulation 1986; 74: 230-238.

45 Ryan T, Petrovic O, Dillon JC, et al. An echocardiographic index for separation of right ventricular volume and pressure overload. J Am Coll Cardiol 1985; 5: 918-927.

46 Abbas AE, Fortuin FD, Schiller NB, et al. A simple method for noninvasive estimation of pulmonary vascular resistance. J Am Coll Cardiol 2003; 41: 1021-1027.

47 Scapellato F, Temporelli PL, Eleuteri E, et al. Accurate noninvasive estimation of pulmonary vascular resistance by Doppler echocardiography in patients with chronic failure heart failure. J Am Coll Cardiol 2001; 37: 1813-1819.

48 Galiè N, Hinderliter AL, Torbicki A, et al. Effects of the oral endothelin-receptor antagonist bosentan on echocardiographic and Doppler measures in patients with pulmonary arterial hypertension. J Am Coll Cardiol 2003; 41: 1380-1386.

49 Tei C, Ling LH, Hodge DO, et al. New index of combined systolic and diastolic myocardial performance: a simple and reproducible measure of cardiac function - a study in normals and dilated cardiomyopathy. J Cardiol 1995; 26: 357-366.

50 Roberts JD, Forfia PR. Diagnosis and assessment of pulmonary vascular disease by Doppler echocardiography. Pulm Circ 2011; 1: 160-181.

51 Grünig E, Weissmann S, Ehlken N, et al. Stress Doppler echocardiography in relatives of patients with idiopathic and familial pulmonary arterial hypertension: results of a multicenter European analysis of pulmonary artery pressure response to exercise and hypoxia. Circulation 2009; 119: 1747-1757.

52 Sachdev A, Villarraga HR, Frantz RP, et al. Right ventricular strain for prediction of survival in patients with pulmonary arterial hypertension. Chest 2011; 139: 1299-1309.

53 Hammarstrom E, Wranne B, Pinto FJ, et al. Tricuspid annular motion. J Am Soc Echocardiogr 1991; 4: 131-139.

54 Lopez-Candales A, Dohi K, Rajagopalan N, et al. Defining normal variables of right ventricular size and function in pulmonary hypertension: an echocardiographic study. Postgrad Med J 2008; 84: 40-45. 